\title{
DISCOVERY OF THE SIXTH DBV STAR: CBS 114
}

\author{
D. E. Winget* and C. F. Claver \\ McDonald Observatory and Department of Astronomy, \\ The University of Texas at Austin
}

Though widely spread across the H-R diagram the compact pulsators have much in common. All are multi-periodic, and most have extremely complex light curves. All appear to be pulsating in nonradial g-modes, with temperature variations responsible for the bulk of the light modulations. The g-modes are global in nature and, typically, many are excited in each pulsator, so they are a rich source of seismological information about the interior regions of the white dwarf stars.

The pulsating DB white dwarf stars (DBV's) form one of the three distinct classes of pulsating compact ob jects currently known. We find these classes nearly uniformly distributed in $\log T_{e}$ spanning virtually the whole range of the white dwarf cooling sequence, from the hot DOV stars at $\log T_{e} \sim 5$ to the DAV (ZZ Ceti) stars at $\log T_{e} \sim 4$; the DBV stars, with $\log T_{e} \sim 4.5$, fall in the middle.

In this paper we present the discovery of pulsations in the DB white dwarf CBS114 (Wagner, et al. 1988, Pesch and Sanduleak 1986), thereby making this object the 6th known DBV.

The light curves of our two runs on this object are shown in Figure 1. These data were obtained with the $2.1 \mathrm{~m}$ telescope at McDonald Observatory using unfiltered light and a blue-sensitive (RCA 8850) phototube. The effects of extinction, as well as long time-scale transparency variations, have been removed by dividing the data by a best fit 3rd order polynomial after sky- subtraction.

The peak-to-peak amplitude of the pulsations in this object are about $0.3 \mathrm{mag}$ and have a quasiperiod of about $650 \mathrm{~s}$. The Fourier transforms of the light curves are shown in Figure 2. These transforms readily demonstrate the complex nature of the light curve: more than 20 statistically significant peaks occur in each of the runs. The power appears in a series of frequency bands, with the largest amplitude band centered on a period of about $650 \mathrm{~s}$ - the quasiperiod evident in the light curve. These results are typical of 5 of the 6 known DBV stars-the exception is $P G 1351+489$ (cf. Winget 1988) with its nearly monoperiodic structure.

The power is distributed in bands of power evenly spaced in frequency, as is also typical of the DBV stars. Thus the bands are not simple harmonics of the large amplitude groups - a pattern one might expect from such a high amplitude, presumably nonlinear, pulsator. The large number of frequencies present in most of the DBV stars indicates that the band-pass of the mode selection mechanism is rather broad, particularly as compared to the bulk of the ZZ Ceti stars. This is consistent with the modetrapping theory (cf. Bradley et al. these proceedings). Unfortunately this kind of loose correlation is as far as we can go with the current observational data.

Since the light curves of none of the complex DBV stars has been completely resolved, the relations between the frequencies of the pulsations are unknown, so a comparison (beyond the simple search for harmonics) with even the simplest nonlinear theories is not currently possible. Therefore, until extended

* Alfred P. Sloan Fellow 

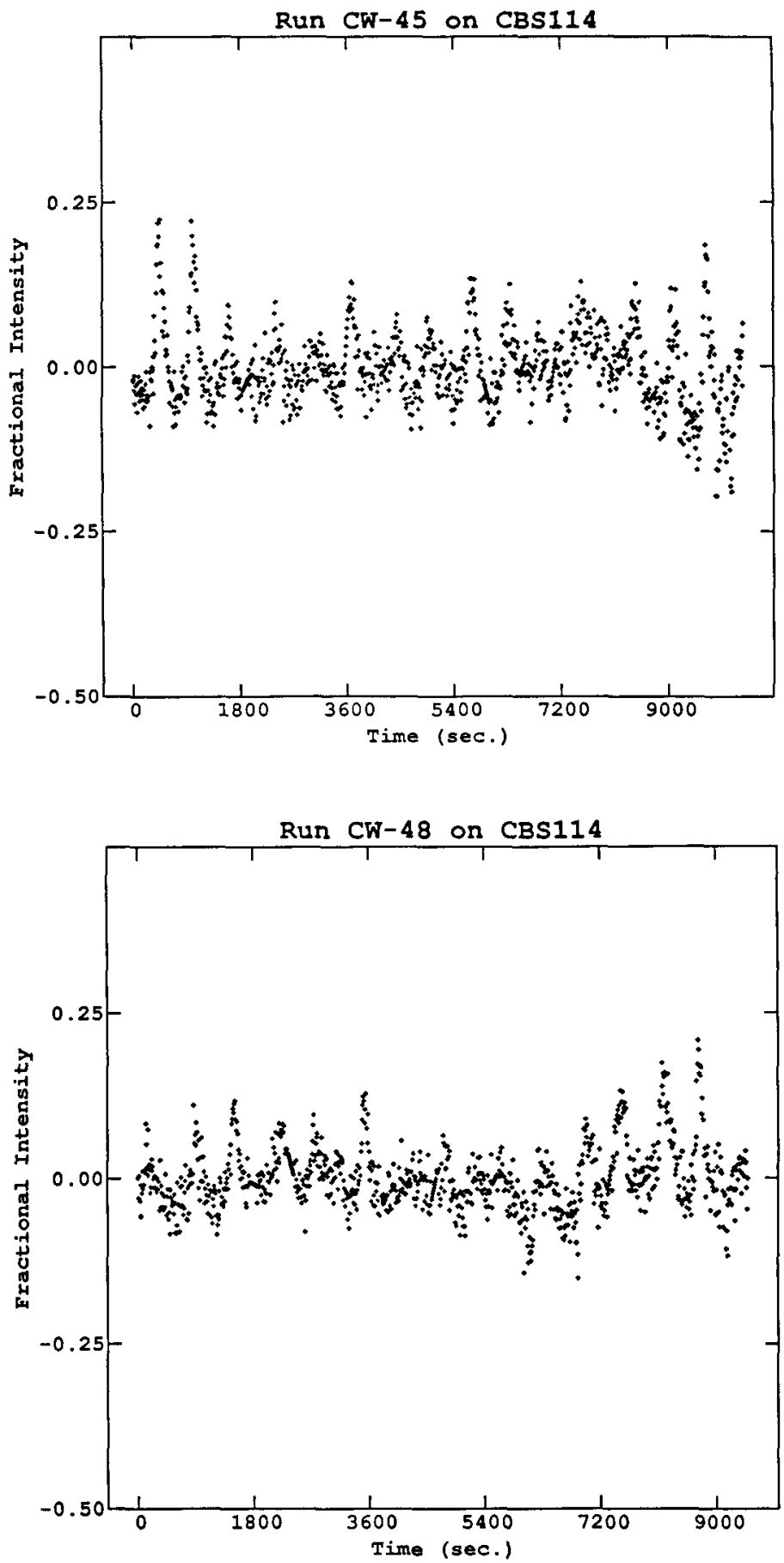

Figure 1 
time-base data is obtained for these objects the seismological information contained in the rich structure of their pulsations will remain hidden.

This work was supported in part by grants, AST 85-52457 and AST 86-00507, from the National Science Foundation.

\section{References}

Pesch, P., and Sanduleak, N. 1986, Ap. J. Suppl, 60, 543 .

Wagner, R. M., Sion, E. M., Liebert, J., Starrfield, S. G. 1988, Ap. J., 328, 213.

Winget, D. E., Seismological Investigations of Compact Stars, in Advances in Helio- and Asteroseismology, eds. J. Christensen and S. Frandsen (Copywrite 1988 by the IAU.).
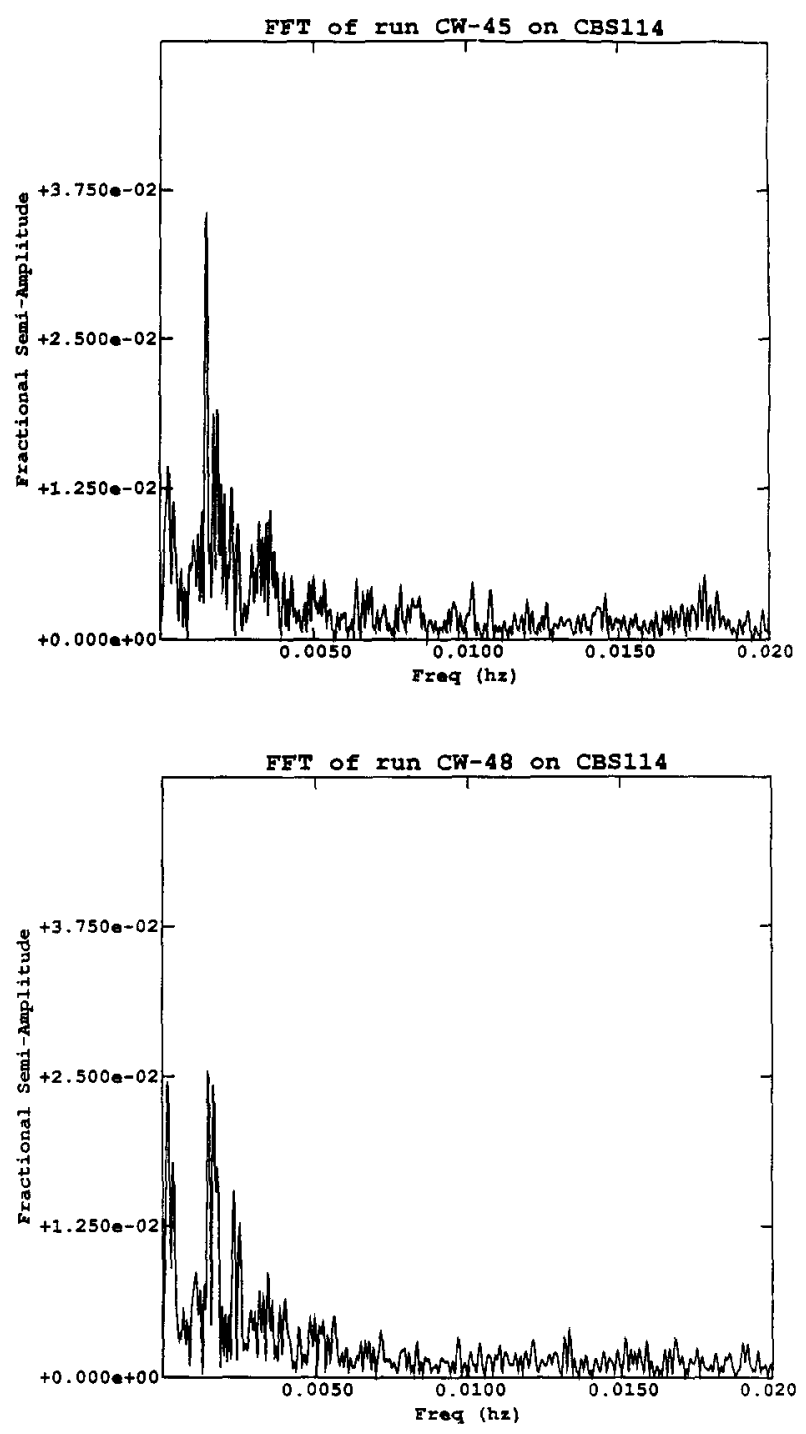

Figure 2 